\title{
Decompressive craniectomy, ICP monitoring and secondary necrosectomy as treatment options in patients presenting with malignant ischemic infarctions extending beyond the middle cerebral artery territory
}

\author{
Niklas Marklund ${ }^{1}$ (D) \\ Received: 6 September 2017 / Accepted: 8 September 2017 /Published online: 30 September 2017 \\ (C) Springer-Verlag GmbH Austria 2017
}

The term "malignant" is commonly used for large spaceoccupying middle cerebral artery (MCA) infarcts. They comprise $1-10 \%$ of all supratentorial strokes, may be more common in females and typically present at a younger age compared to other forms of ischemic stroke [1]. In the infarcted brain regions, a profound brain edema develops resulting in midline shift, compression of the basal cisterns and increased intracranial pressure (ICP). Using best medical management, mortality exceeds $80 \%$ in most clinical series. Decompressive craniectomy (DC), i.e., removal of a large bone flap over the infarcted area combined with duraplasty, alleviates the increase in ICP and may prevent brain herniation. Thus, DC is commonly life-saving in selected malignant MCA infarct cases. However, in view of the devastating underlying brain injury and fear of intolerable neurological deficits in DC survivors, this surgery was only infrequently performed before the 1990s. Then, the influential mid-2000s randomized controlled trials (RCTs) DECIMAL, DESTINY and HAMLET were able to show that DC markedly reduced mortality without increasing the risk of leaving the survivor in a most severely disabled state in patients younger than 60 years old and surgically treated within $48 \mathrm{~h}$ of stroke onset [2]. The case fatality reduction by DC was estimated to be ca. $50-75 \%$, and these RCTs substantially changed neurosurgical practice. To date, an ever increasing number of publications have documented the role of DC in malignant MCA infarct patients, addressing not only the potential clinical benefits but also complications and important ethical and quality of life

Niklas Marklund

niklas.marklund@med.lu.se

1 Department of Clinical Sciences Lund, Neurosurgery, Lund University, Skåne University Hospital, Lund, Sweden concerns [3]. However, there are some remaining clinical questions regarding malignant MCA infarcts that have been insufficiently addressed in the literature.

- Is there also a role for DC in patients with malignant MCA infarcts presenting with additional involvement of the anterior and/or posterior cerebral artery territory, the MCA+ infarcts?

- Is postoperative ICP monitoring and neurocritical care indicated following DC?

- In the event of an increased ICP and/or increased midline shift despite a DC of adequate size, is there an indication for additional surgical therapy using necrosectomy, i.e., surgical removal of infarcted areas?

These important clinical questions were retrospectively addressed in the present issue of Acta Neurochirurgica by Kürten and colleagues in Germany. They analyzed $101 \mathrm{ma}-$ lignant MCA infarcts patients, 27 of whom had an MCA+ infarcts. All included patients underwent DC, and outcome measures were Glasgow Coma Scale (GCS) score at 14 days and the modified Rankin Scale (mRS) score at 3 months postsurgery. ICP was measured postoperatively in 44 patients, and 20 required necrosectomy because of persisting midline shift and/or increased ICP. The authors confirmed that high age and a depressed level of consciousness at the time of DC are negative prognostic factors in malignant MCA infarcts.

In the $27 \mathrm{MCA}+$ patients, the most common additional vascular territory involved was the anterior cerebral artery $(63 \%, n=17)$. At 3 months post-surgery, $26 \%$ reached an mRS of 2-3 and $48 \%$ had an mRS $=4$, meaning that two thirds of these patients had a moderate-severe disability. Importantly, clinical outcomes in MCA+ infarct patients were similar to those of infarcts confined to the MCA territory, implying that the additional severity of the underlying brain 
injury did not translate into a worse outcome for the patient. However, the GCS prior to DC, age and midline shift were not presented separately for the MCA+ cohort, and it is unclear whether a selection bias may have existed for surgery because of fewer negative prognostic factors at baseline. Obviously, in view of the extent of the infarcted brain areas in MCA and $\mathrm{MCA}+$ infarctions, full recovery cannot be expected, and most survivors are left with persistent and profound neurological deficits influencing their quality of life. In most neurosurgical disorders, an outcome of mRS 4 (unable to walk or attend to his/her own bodily needs without assistance) cannot be considered a good or desired outcome. Whether an mRS of 4 is an acceptable outcome in terms of quality of life and rate of disability remains a topic of debate. It should be remembered that in most studies of DC survivors, quality of life and the retrospective consent to treatment is surprisingly high [4]. The decision to perform or refrain from DC should be influenced by many factors including the views of the patient and/or his/ her family, biological age and comorbidities of the patient, although the data presented here suggest that the presence of an MCA+ infarct is not one of these factors excluding the patient from surgical treatment.

The authors also describe their postoperative neurocritical care strategy. Following DC, all patients remained under anesthesia and were then evaluated by a routine postoperative CT scan and sedation interruption for clinical assessment. The authors used an intraventricular catheter for ICP monitoring in 44 patients presenting with preoperative ventricular dilatation and/or a decreased level of consciousness. ICP-monitored patients had a less favorable outcome, not surprising in view of their worse preoperative clinical situation and that the indication for ICP monitoring was linked to a severe neurological condition prior to DC. However, ICP elevation per se was not associated with a less favorable outcome in those monitored. The ICP monitoring was performed only in selected patients, and it is plausible that the knowledge of ICP dynamics could help identify patients requiring additional therapies. Further studies should clarify whether postoperative ICP monitoring and neurocritical care are justified in selected patients [5-6] and whether a more active management of patients with increased ICP following DC can positively influence outcome.

Occasionally, an adequate-size DC [7] will not sufficiently alleviate the infarct-induced mass effect, leaving the treating neurosurgeon with the dilemma of determining whether additional surgery is worthwhile. In the present study, 20 patients with a remaining midline shift of an average $>12 \mathrm{~mm}$ and/or increased ICP following DC underwent a second-look procedure where clearly demarcated and infarcted tissue at the frontal and/or temporal lobes was removed. Eleven of these 20 patient had an MCA+ infarction. In view of the extent of the underlying brain injury, it is not surprising that necrosectomy patients had an approximately $25 \%$ worse outcome reflected by a lower GCS on postoperative day 14 and at 3-month follow-up. However, three patients reached an mRS of 2-3 and nine an mRS of 4 at 3 months post-surgery with an overall rate of moderate-severe disability of $60 \%$, suggesting that necrosectomy may be a valid additional treatment option using careful selection criteria.

The DESTINY-II trial [8] convincingly showed that DC markedly reduced mortality also in the elderly, $60-80$ years old, patients. However, the functional recovery was substantially worse in these elderly patients than that of younger patients, and the decision to perform DC in an elderly patient remains controversial. Since $40 \%$ of all malignant MCA infarct patients are $>60$ years, such considerations are clinically important. In this article by Kürten et al., the mean age was 56 years old with the oldest patient being 79 years old. Clearly, the age of 60 suggested by previous RCTs [2] was not used as a strict cutoff for DC in the present study. These results confirmed that high age is a negative prognostic factor, and the age of the patient should carefully be considered in the decision whether to perform DC in both MCA and MCA+ infarctions.

Since these data are retrospective, the results must be treated with caution. Moreover, the number of patients with $\mathrm{MCA}+$ infarcts is small, arguing that robust conclusions at a high level of scientific evidence cannot be made. Yet, these data provide important information for the neurosurgeon evaluating a patient with an MCA+ infarct and suggest that postoperative ICP monitoring and secondary necrosectomy may have a role in MCA/MCA+ patients selected by strict criteria. The most important contribution of the paper by Kürten et al. is that the results of DC in MCA+ patients were similar to those of MCA patients. Thus, DC could also be considered in patients with infarcts extending beyond the MCA territory. DC territory, even though a large infarct volume is a negative prognostic factor [9]. However, the provided data do not imply that a larger proportion of malignant MCA infarct patients should be subjected to DC. Instead, the same clinical judgment and ethical considerations used for a patient with a "regular" malignant MCA infarct should also apply to patients with infarcts extending beyond the MCA territory. More detailed quality of life data and neuropsychological outcome measures would be welcome in future studies to further guide the clinician treating severely brain-injured patients presenting with MCA+ infarctions.

\section{References}

1. Heiss WD (2016) Malignant MCA infarction: pathophysiology and imaging for early diagnosis and management decisions. Cerebrovasc Dis 4:1-7

2. Vahedi K, Hofmeijer J, Juettler E, Vicaut E, George B, Algra A, Amelink GJ, Schmiedeck P, Schwab S, Rothwell PM, Bousser MG, van der Worp HB, Hacke W, DECIMAL, DESTINY, and HAMLET investigators (2007) Early decompressive surgery in 
malignant infarction of the middle cerebral artery: a pooled analysis of three randomised controlled trials. Lancet Neurol 6(3):215-222

3. McKenna A, Wilson FC, Caldwell S, Curran D, Nagaria J, Convery F (2012) Long-term neuropsychological and psychosocial outcomes of decompressive hemicraniectomy following malignant middle cerebral artery infarctions. Disabil Rehabil 34:1444-1455

4. Rahme R, Zuccarello M, Kleindorfer D, Adeoye OM, Ringer AJ (2012) Decompressive hemicraniectomy for malignant middle cerebral artery territory infarction: is life worth living? J Neurosurg 117:749-754

5. Paldor I, Rosenthal G, Cohen JE, Leker R, Harnof S, Shoshan Y, Itshayek E (2015) Intracranial pressure monitoring following decompressive hemicraniectomy for malignant cerebral infarction. J Clin Neurosci 22:79-82

6. Sauvigny T, Göttsche J, Czorlich P, Vettorazzi E, Westphal M, Regelsberger J (2017) Intracranial pressure in patients undergoing decompressive craniectomy: new perspective on thresholds. J Neurosurg 14:1-9

7. Johnson RD, Maartens NF, Teddy PJ (2011) Technical aspects of decompressive craniectomy for malignant middle cerebral artery infarction. J Clin Neurosci 18:1023-1027

8. Jüttler E, Unterberg A, Woitzik J, Bösel J, Amiri H, Sakowitz OW, Gondan M, Schiller P, Limprecht R, Luntz S, Schneider H, Pinzer T, Hobohm C, Meixensberger J, Hacke W, DESTINY II Investigators (2014) Hemicraniectomy in older patients with extensive middle-cerebral-artery stroke. N Engl J Med 370: 1091-1100

9. Hecht N, Neugebauer H, Fiss I, Pinczolits A, Vajkoczy P, Jüttler E, Woitzik J (2017) Infarct volume predicts outcome after decompressive hemicraniectomy for malignant hemispheric stroke. J Cereb Blood Flow Metab 1. doi:10.1177/ $0271678 X 17718693$ 